\title{
Simplified adaptive linear neuron harmonics extraction algorithm for dynamic performance of shunt active power filter
}

\begin{abstract}
This paper presents a single-phase shunt active power filter with artificial intelligence based algorithms by introducing a new Simplified Adaptive Linear Neuron (ADALINE) algorithm for harmonics extraction. The proposed harmonics extraction is an innovative work over the established algorithm, known as Modified Widrow-Hoff ADALINE algorithm. Three significant major improvements are made by modifying weight updating technique which leads to introduction of fundamental active current updating technique, removing cosine component according to symmetrical theory of periodic signal, and minimizing large average square error by removing sum of elements. The SAPF with the proposed harmonics extraction algorithm was simulated in MATLAB/Simulink. For practical validation, the experimental laboratory prototype was also developed, and the algorithm was implemented using Digital Signal Processor TMS320F28335. Both simulation and experimental tests cover different types of nonlinear loads and dynamic operation of loads. From simulation and experimental results, significant improvements in terms of Total Harmonic Distortion and response time are shown together with comparison analysis with the established algorithm.
\end{abstract}

Keyword: Shunt active power filter; Artificial neural network; Adaptive Linear Neuron; Power quality; Current harmonic; Total Harmonic Distortion 